\title{
Une pyélonéphrite emphysémateuse d'évolution favorable après traitement médical : A propos d'un cas.
}

\section{An emphysematous pyelonephritis of favorable evolution after medical treatment:} About a case

\author{
Fongoro S, Fofana AS, Coulibaly M, Yattara H, Djiguiba K, Diallo D, Coulibaly SB, Samaké M. \\ Service de néphrologie et d'hémodialyse du CHU du point G, Bamako/Mali \\ E-mail de l'auteur : saharefongoro@yahoo.fr
}

\section{Résumé :}

Introduction : La pyélonéphrite emphysémateuse est une infection nécrotique du rein caractérisée par la présence de gaz au sein du parenchyme rénal, des cavités excrétrices ou des espaces péri-rénaux. Objectif : Notre propos est de montrer l'efficacité du traitement médical, surtout au stade précoce de l'affection. Observation : Nous rapportons un cas de pyélonéphrite emphysémateuse chez une femme de 60 ans, diabétique, dont l'évolution a été favorable sous antibiotique sans en avoir recours à la chirurgie. Commentaire: Décrite pour la première fois en 1898, la pyélonéphrite emphysémateuse est une infection grave engageant le pronostic vital. Affection rare, survenant préférentiellement sur terrain diabétique, est liée au développement de germes anaérobies. Elle peut se compliquer d'un choc septique et d'une défaillance multi viscérale. La tomodensitométrie est l'examen de référence qui permet le diagnostic positif par la présence de gaz au sein du parenchyme rénal, des cavités excrétrices ou des espaces péri-rénaux. Les attitudes thérapeutiques divergent entre traitement chirurgical, drainage percutané et traitement conservateur. Conclusion : Cette observation illustre la place du traitement médical au stade précoce de la maladie.

Mots clés : Diabète, pyélonéphrite emphysémateuse, antibiothérapie.

\begin{abstract}
Introduction: Emphysematous pyelonephritis is a necrotic kidney infection characterized by the presence of gas within the renal parenchyma, excretory cavities or perirenal spaces. Aim: Our purpose is to show the effectiveness of medical treatment, especially at the early stage of the disease. Case report: We report a case of emphysematous pyelonephritis in a diabetic 60year-old woman who had a favorable course of antibiotics without the need for surgery. Comment: Described for the first time in 1898, emphysematous pyelonephritis is a serious and lifethreatening infection. Rare disorder, occurring preferentially on diabetic ground, is related to the development of anaerobic germs. It can be complicated by septic shock and multi-visceral failure. Computed tomography is the reference
\end{abstract}

examination that allows positive diagnosis by the presence of gas in the renal parenchyma, excretory cavities or perirenal spaces. Therapeutic attitudes differ between surgical treatment, percutaneous drainage and conservative treatment. Conclusion: This observation illustrates the place of medical treatment at the early stage of the disease.

Key words: Diabetes, emphysematous

pyelonephritis, antibiotic therapy.

\section{Introduction :}

Décrite initialement en 1898, la pyélonéphrite emphysémateuse (PNE) est une infection nécrotique du rein caractérisée par la présence de gaz au sein du parenchyme rénal, des cavités excrétrices ou des espaces perirénaux [1]. C'est une affection grave engageant rapidement le pronostic vital par état de choc septique et survenant préférentiellement chez les diabétiques déséquilibrés aussi bien insulino que non insulinodépendants. Les signes cliniques ne sont pas spécifiques conduisant au retard diagnostique. La tomodensitométrie (TDM) est l'examen de référence et permet d'élucider le diagnostic, d'évaluer le pronostic et décider de l'attitude thérapeutique et propose une classification en quatre stades pronostiques. Les attitudes thérapeutiques divergent entre traitement mutilant, drainage percutané et traitement conservateur. Notre observation illustre la place du traitement médical dans les formes précoces.

Observation :

Il s'agissait d'une patiente de 60 ans, connue diabétique et hypertendue. Elle a été hospitalisée le 07/09/2016 dans le service de néphrologie du CHU $\mathrm{du}$ point $\mathrm{G}$ pour masse du flanc gauche. L'interrogatoire retrouvait l'apparition d'une douleur lombaire gauche à type de pesanteur sans irradiation depuis deux semaines environ. Elle associait une asthénie, une anorexie et des vertiges. On retrouvait également des troubles de la miction à type de pollakiurie et de brûlures mictionnelles. L'examen général notait une pression artérielle à $150 / 90 \mathrm{mmHg}$, une fréquence cardiaque à 90 battements/min, le poids à $45 \mathrm{Kg}$ avec l'index de masse corporel (IMC) à $16,2 \mathrm{Kg} / \mathrm{m}^{2}$, et la température à $37,8^{\circ} \mathrm{C}$. La palpation de l'abdomen retrouvait une masse douloureuse ferme avec 
contact lombaire du flanc gauche. Ailleurs l'examen clinique était sans particularité. Le bilan biologique à l'admission révélait une hyperleucocytose à $20300 / \mathrm{mm}^{3}$ à prédominance polynucléaires neutrophiles $\left(14800 \mathrm{~mm}^{3}\right)$, une CRP à $143,4 \mathrm{mg} / \mathrm{l}$, une procalcitonine à $13 \mathrm{ng} / \mathrm{ml}$, une créatininémie à $87 \mu \mathrm{mol} / 1$, une urée sanguine à 3 $\mathrm{mmol} / \mathrm{l}$, une glycémie à $10,4 \mathrm{mmol} / \mathrm{l}$ et une hémoglobine glyquée à $12 \%$. La culture bactérienne des urines a révélé un Escherichia coli sensible à la ceftazidim. La sérologie de l'hépatite B, C et du VIH était négative. La radiographie thoracique de face sans particularité. Devant ce tableau clinicobiologique, un scanner abdominal réalisé d'emblée a montré un gros rein gauche $(155 \times 78 \times 74 \mathrm{~mm})$ à contour régulier siège d'une collection multicloisonnée à paroi épaisse et régulière, à contour hétérogène $(39 \times 30 \mathrm{~mm})$ du pôle supérieur.

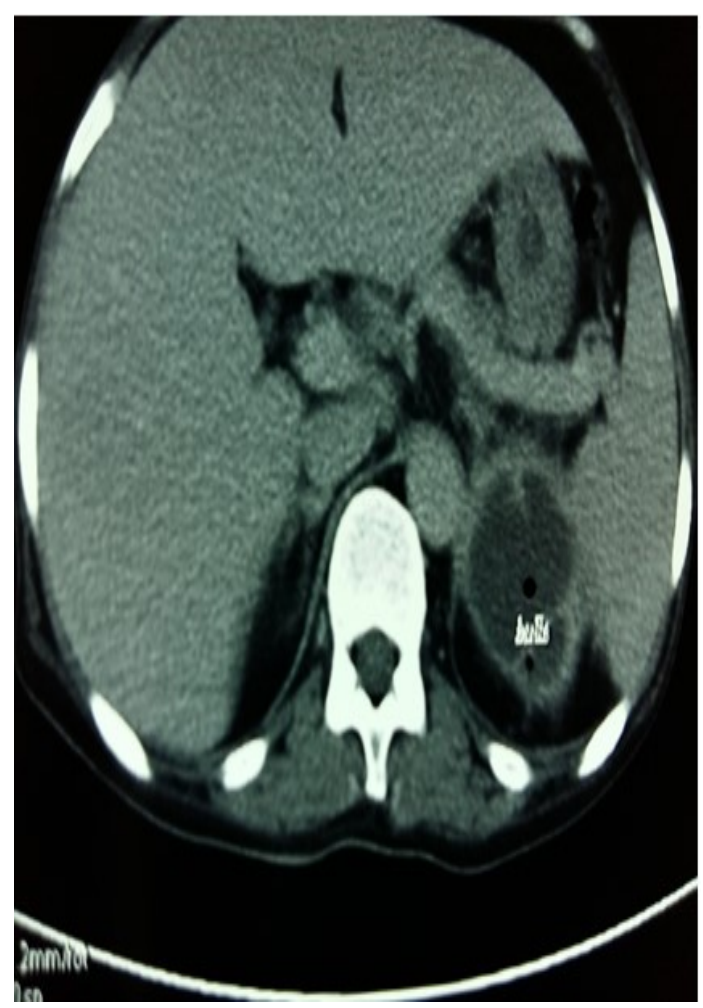

Figure 1 : Présence de bulles d'aires dans le rein gauche

\section{Discussion :}

La pyélonéphrite emphysémateuse est une complication rare des infections du tractus urinaire pouvant mettre en jeu le pronostic vital (2). Elle est caractérisée par une atteinte gazeuse d'origine bactérienne du parenchyme rénal avec un risque d'extension vers les organes s'étendant à travers la capsule rénale vers l'espace peri-nephretique (3). Son incidence est en augmentation en raison d'une
Il s'y associait une infiltration de la graisse périnéale, une importante dilatation des cavités pyélocalicielles à contenu hétérogène renfermant une macrobulle de gaz sans extension, aspect évocateur d'une pyélonéphrite emphysémateuse de stade $3 \mathrm{~A}$. Le rein droit était sans particularité $(114 \times 51 \times 57 \mathrm{~mm})$. Un traitement antibiotique à base de ceftazidim en association au métronidazole a été démarré par voie parentérale. Ce traitement a été poursuivi pendant trois semaines avec retour à la normal des paramètres biologiques (Tableau 1). A défaut de l'uroscanner de contrôle pour raison de coût, une échographie rénale réalisée deux semaines après le début du traitement montrait des reins de taille normale mesurant $113 \times 58 \mathrm{~mm}$ à droite et $113 \times 51 \mathrm{~mm}$ à gauche.

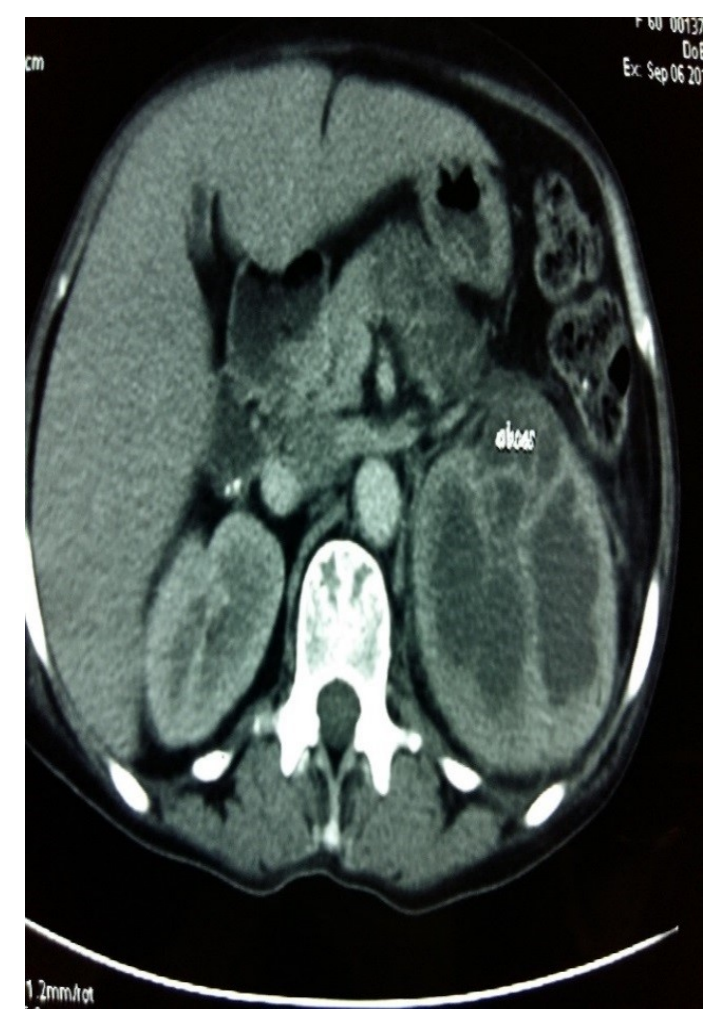

Figure 2 : collection multicloisonnée à paroi épaisse et régulière, à contour hétérogène $\mathrm{du}$ pôle supérieur + infiltration de la graisse périnéale

meilleure connaissance de la maladie, de la diffusion de la tomodensitométrie ou de l'augmentation de l'incidence du diabète en Asie et dans les pays industrialisés. En effet, il s'agissait de patients diabétiques dans plus de $90 \%$ des cas, plus volontiers d'une forme mal équilibrée (4). La plupart des cas rapportés dans la littérature étaient sporadiques, la plus grande série étant celle de Wane (5) à propos de 38 cas. Dans notre cas il 
s'agissait d'une forme unilatérale chez une femme âgée diabétique mal équilibrée avec une importante dilatation utero-pyélocalicielle du rein gauche. Un obstacle à l'écoulement de l'urine, extrinsèque ou intrinsèque est montré dans 20 à $40 \%$ des cas. La maladie a débuté par une infection du rein par un germe gazogène, exceptionnellement anaérobie strict. L'Escherichia coli est isolé dans les urines ou dans le sang dans plus de la moitié des cas (6).

La principale hypothèse de la production de gaz est celle de la fermentation intra rénale du glucose. La glycolyse par la voie d'Embden Meyerhof aboutit à la production de Pyruvate. Sur le plan clinique, la triade associant un diabète sucré, une infection urinaire et une obstruction est évocatrice du diagnostic. Les symptômes sont ceux d'une pyélonéphrite aiguë ou d'un abcès rénal avec fièvre, frissons, nausées et vomissements. La neuropathie diabétique peut retarder le diagnostic en réduisant la symptomatologie douloureuse et favorise la survenue de formes graves (7). Le tableau biologique dans notre cas montrait une hyperleucocytose $\left(20300 / \mathrm{mm}^{3}\right)$, une hyperglycémie $(10,45 \mathrm{mmol} / \mathrm{l})$, une accélération de la vitesse de sédimentation, de la protéine $\mathrm{C}$ réactive $(143,41$ $\mathrm{mmol} / \mathrm{l}$ ) et une procalcitonine à $13 \mathrm{ng} / \mathrm{ml}$. Il n'y avait pas d'altération de la fonction rénale et de trouble de la coagulation en rapport avec un sepsis grave chez notre patiente. La tomodensitométrie est l'examen de référence pour le diagnostic et le suivi de la PNE, permettant une localisation précise du siège du gaz dans le parenchyme rénal ou le système collecteur, ou dans l'espace péri néphrétique (8). Huang et Tseng ont établi une classification radiologique à valeur pronostique, conditionnant le choix thérapeutique (9)

- Stade 1: gaz dans les voies excrétrices seulement

- Stade 2: gaz dans le parenchyme rénal sans extension dans l'espace extra-rénal

- Stade $3 \mathrm{~A}$ : extension de gaz ou abcès de l'espace perinéphretique

- Stade 3B : extension de gaz ou abcès de l'espace pararénal

- $\quad$ Stade 4 : PNE bilatérale ou sur rein unique. Notre cas correspond au stade $3 \mathrm{~A}$ de la classification radiologique de Huang et Tseng. Dans notre cas il n'y avait pas eu de radiographie de l'arbre sans préparation pouvant révélé l'emphysème rénal dans $85 \%$ des cas ou un pneumopéritoine. Il en était de même pour l'échographie qui pouvait montrer l'ombre rénal masqué par l'interposition de gaz au stade avancé, mais l'échographie réalisée deux semaines après l'antibiothérapie à défaut du scanner faute de moyens financier montrait des reins sans particularité mesurant $113 \times 58 \mathrm{~mm}$ à droite et $113 \times 51 \mathrm{~mm}$ à gauche. La PNE est une urgence thérapeutique. En dehors des troubles hémodynamiques, hydro électrolytiques et des dysfonctions d'organe, le traitement étiologique a reposer sur l'option d'antibiothérapie simple sans drainage percutané ni néphrectomie dans notre cas après analyse répété de la tomodensitométrie et de la sévérité de l'état clinique du patient.

Conclusion : La PNE est une infection rare du parenchyme rénal mettant en jeu le pronostic fonctionnel et vital par la survenu d'un choc septique. Le scanner participe à la confirmation diagnostique et l'évaluation pronostique et thérapeutique de cette affection. Son traitement est actuellement de plus en plus médical surtout en absence de facteur de mauvais pronostic.

Conflit d'intérêt : Les auteurs ne déclarent pas de conflit d'intérêt.

Références:

1. Kelly. H A; Mc Callum W G. Pneumaturia. JAMA 1898; 31: 375-82

2. Turman A E; Rutherford C. Emphysematous pyelonephritis with perinephretic gas. J urol 1971; 105: $165-70$

3. Kably.MI, EImrou.F,Chikhaoui.N. Emphysematous pyelonephritis: radiologic diagnostis, Am Urol 2003; 37(10): 229-32

4. Micheali. J; Mogle.P, Perlverg. S Emphysematous pyelonephritiw. J Urol 1984; 13(2) 203-8

5. Wane Y L; LO. S.K; Bullard.M.J; Chanfg.P.L; Lec. T Y. Predictors of outcome in emphysematous pyelonephritis. J Urol 1998; 159: 369-73

6. Jacobson. S H; Ostenson. C G; Tullus.K; Brauner. A. Serum resistance in Escherichia coli strains causing acute pyelonephritis and bacteriama. APMIS 1992, 100(2):147-53

7. Guillausseau. PJ; Farah.R; Laloi Michelin. M; Tielmans . A; Rymer.R ; Warnet.A. Urinary tract infection in patients with diabetes mellitus tract infections in patients with diabetes melletus (infection urinaire et diabete sucré). Rev Prat 2003; 53(16): 1790-6

8. Kaiser.E; Fournier R. Emphysematous pyelonephritis diagnosis and traitement. Ann Urol 2005; 39:49-60.

9- Huang JJ, Tseng CC. Emphysematous pyelonephritis: Clinicoradiological classification, management, prognosis and pathogenesis. Arch.Intern.Med. 2000;160(6):797-805. 
Tableau 1 : Paramètres biologiques

\begin{tabular}{|l|l|l|}
\hline Paramètres & A l'admission & Contrôle \\
\hline Leucocytes & $\mathbf{2 0 3 0 0} / \mathbf{m m}^{\mathbf{3}}$ & $9200 / \mathrm{mm}^{3}$ \\
Hémoglobine & $8,2 \mathrm{~g} / \mathrm{dl}$ & $11,7 \mathrm{~g} / \mathrm{dl}$ \\
Plaquettes & $314000 / \mathrm{mm}^{3}$ & $442000 / \mathrm{mm}^{3}$ \\
CRP & $\mathbf{1 4 3 , 4 1} \mathbf{~ m g} / \mathbf{l}$ & $0,57 \mathrm{mg} / 1$ \\
Procalcitonine & $\mathbf{1 3} \mathbf{~ n g} / \mathbf{m l}$ & $0,17 \mathrm{ng} / \mathrm{ml}$ \\
Glycémie & $10,45 \mathrm{mmol} / 1$ & $5,5 \mathrm{mmol} / 1$ \\
HbA1C & $12 \%$ & $8 \%$ \\
Protéinurie de $24 \mathrm{H}$ & $560 \mathrm{mg} / 24 \mathrm{H}$ & $312 \mathrm{mg} / 24 \mathrm{H}$ \\
Leucocyturie & $\mathbf{2 2 3 0 0} / \mathbf{m l}$ & $<10000 / \mathrm{ml}$ \\
Uroculture & Escherichia coli & Stérile \\
Créatininémie & $87 \mu \mathrm{mol} / 1$ & $73 \mu \mathrm{mol} / 1$ \\
Urée sanguine & $3 \mathrm{mmol} / \mathrm{l}$ & $3,8 \mathrm{mmol} / 1$ \\
\hline
\end{tabular}

